\title{
Pancoast's syndrome due to chronic pneumonia
}

\author{
J. Ribas*, L. Lores*, J. Ruiz*, V. Ausina**, J. Morera*
}

Pancoast's syndrome due to chronic pneumonia by Pasteurella multocida. J. Ribas, L. Lores, J. Ruiz, V. Ausina, J. Morera. CERS Journals Ltd 1997.

ABSTRACT: Infectious causes of Pancoast's syndrome are extremely rare. We describe the case of a patient with Pancoast's syndrome due to chronic pneumonia resulting from Pasteurella multocida.

The patient was not immunosuppressed and had had no contact with animals. The diagnosis was made by transthoracic needle aspiration and institution of therapy with cefuroxime-axetil resulted in resolution of his symptoms. Eur Respir J 1997; 10: 2904-2906.
*Dept of Respiratory Medicine and **Microbiology Service, Hospital Universitari "Germans Trias i Pujol", Badalona (Barcelona), Spain.

Correspondence: J. Ribas, Servei de Pneumologia. Hospital Universitari Germans Trias i Pujol, Carretera de Canyet sn., 08916 Badalona, Spain.

Keywords: Pancoast's syndrome, pulmonary pastereurellosis, transthoracic fine needle aspiration

Received: February 191997

Accepted after revision July 51997
In 1932, PANCOAST [1] wrote the original description of the superior pulmonary sulcus tumour. The tumour was characterized by pain, Horner's syndrome, bone destruction and atrophy of hand muscles. Many patients with an apical neoplasm do not fulfil all four criteria, however. The term Pancoast's syndrome is, in fact, now applied to any apical neoplasm that is accompanied by shoulder or arm pain [2].

Infectious causes of Pancoast's syndrome are very rare, and only a few such cases have been reported in the literature. Bacteria of the genus Pasteurella are rarely isolated from the respiratory tract. Pasteurella multocida has been associated with numerous respiratory syndromes, either as a primary or secondary cause of disease [3]. The clinical diagnoses most frequently associated with isolation of $P$. multocida from the respiratory tract are bronchiectasis, bronchogenic carcinoma, chronic obstructive pulmonary disease and pneumonia [3]. P. multocida isolated from the respiratory tract is most commonly a commensal organism in patients with underlying chronic lung disease. Nevertheless, serious infections, such as pneumonia, empyema and lung abscess, may develop $[4,5]$. Most patients with respiratory tract infection or colonization have a history of animal contact [4].

\section{Case report}

A $46 \mathrm{yr}$ old man presented with a 5 month history of nonproductive cough, dyspnoea on exertion, anorexia and $8 \mathrm{~kg}$ weight loss, and sharp pain in his left scapula radiating down the inner side of his left arm. He complained of difficulty sleeping because of pain. He reported no other neurological symptoms.

The patient smoked about 40 cigarettes per day and occasionally drank alcohol. His medical history was unremarkable, except for an appendectomy 10 yrs previously. Chronic bronchitis was not detected by history. The patient denied contact with animals.

On examination, the patient was apyrexial and poorly nourished. There was no clubbing or cyanosis. Decreased inspiratory breath sounds were heard over the upper portion of the left hemithorax. Neurological examination disclosed no abnormality and Horner's syndrome was not detected.

A chest radiograph showed a large opacity in the left upper lobe, with loss of volume (fig. 1a). Sputum cultures did not grow pathogenic bacteria, mycobacteria or fungi, and cytological findings were normal. Full blood count, platelet count and blood chemistry screening were normal. The erythrocyte sedimentation rate was 102 $\mathrm{mm} \cdot \mathrm{h}^{-1}$. There were no biochemical abnormalities suggestive of malnutrition. Pulmonary function testing showed a moderate obstructive ventilatory defect with no response to inhaled bronchodilator (forced expiratory volume in one second (FEV1) 2.49 L, $71 \%$ of predicted value). Fibreoptic bronchoscopy findings were normal, except for a concentric stenosis, with normal mucosa, of the upper division of the left upper bronchus. Cytological examination of bronchial aspirate was normal, and culture did not grow pathogenic bacteria or mycobacteria. Bronchial biopsies showed no neoplastic changes.

Magnetic resonance imaging of the upper thorax (fig. 1b) revealed a left apical mass, with loss of volume. The mass did not respect pleuromediastinal fat and invasion of the superior mediastinum was observed. The patient underwent transthoracic fine needle aspiration of the mass, guided by computed tomography. Again, cytological examination was negative, showing only a mild acute inflammatory reaction consisting mainly of neutrophils. When the material obtained was cultured 
a)
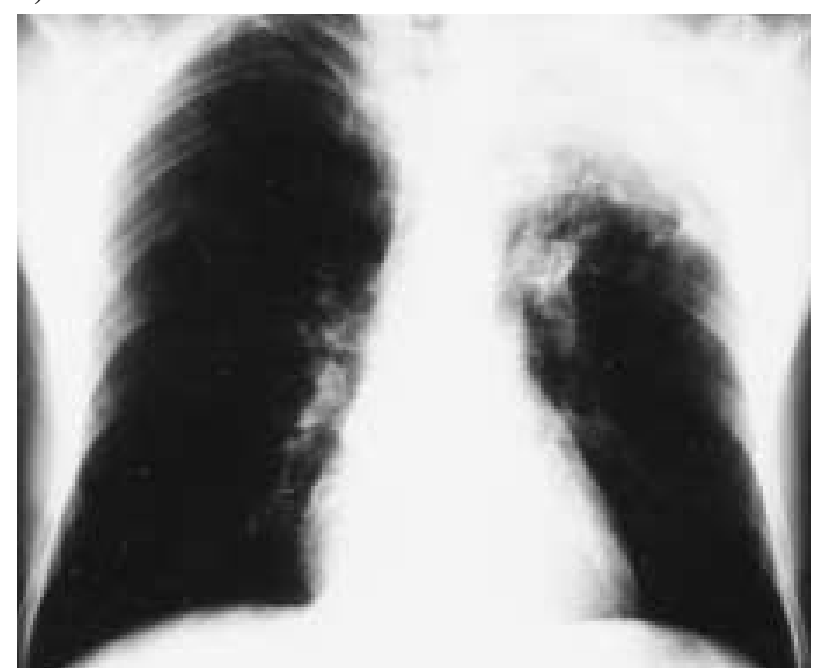

b)

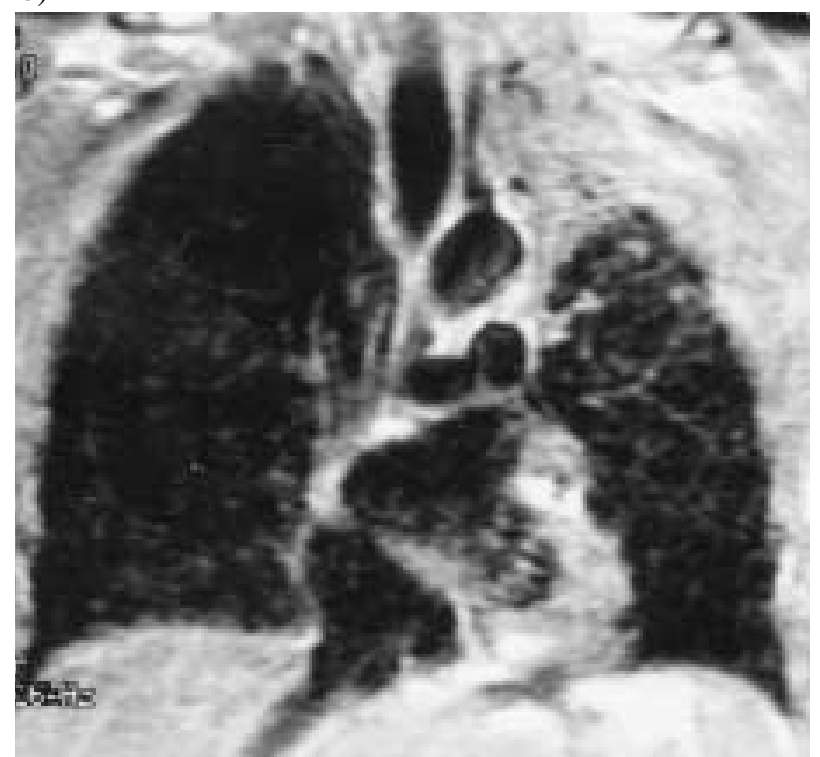

Fig. 1. - a) Chest radiograph at presentation showing left upper lobe consolidation with loss of volume. b) Coronal magnetic resonance image of the upper thorax showing a left apical mass with invasion of the superior mediastinum.

on blood agar, a Gram-negative coccobacillus grew that did not grow on MacConkey medium. The lack of haemolysis on blood agar, the negative urease reaction and the positive indole reaction led to the identification of P. multocida.

Therapy with oral cefuroxime-axetil (500 mg twice daily) was initiated, and the pain disappeared by the third week of treatment. Because of the chronic nature of the process, a long course of antibiotic therapy was advised. Although the first choice antibiotic against $P$. multocida is penicillin, in order to guarantee a better completion of treatment, a drug that could be administered orally twice daily was indicated. Therapy was discontinued after 2 months. By that time, the patient had no symptoms and a chest radiograph showed marked improvement of the left apical mass (fig. 2). After 2 yrs, the patient is doing well and no symptoms have reappeared.
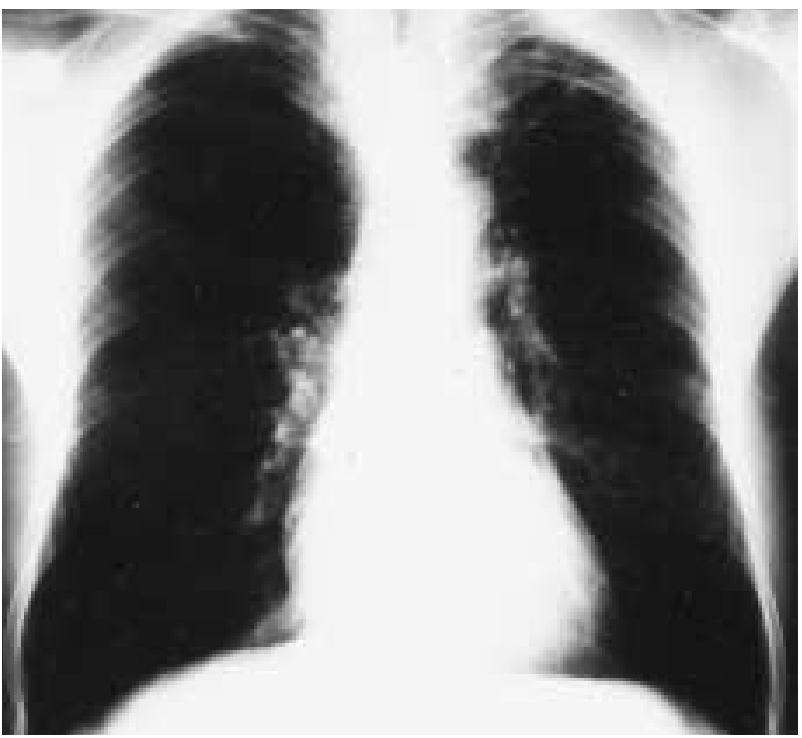

Fig. 2. - Chest radiograph at 2 months of follow-up revealing marked improvement of the left upper lobe consolidation after antibiotic therapy.

\section{Discussion}

Infectious causes of Pancoast's syndrome are extremely rare. Pulmonary tuberculosis can present in this way, as PANCOAST [1] described in his original report. Other infectious causes have rarely been reported in the literature. In 1969, Sтатнатоs et al. [6] described a patient with Pancoast's syndrome due to a hydatid cyst of the thoracic outlet. Fungal infections can also produce nonmalignant Pancoast's syndrome. Aspergillus infection [7, 8] and mucormycosis [9] have been reported as causes of Pancoast's syndrome in patients with haematological malignancies being treated with chemotherapy. Staphylococcus aureus pulmonary infection is another rare but treatable cause of Pancoast's syndrome in nonimmunosuppressed individuals $[10,11]$.

To our knowledge, this is the first reported case of Pancoast's syndrome due to pulmonary pasteurellosis. The negative result of the bacteriological culture of sputum and bronchial aspirate could be explained by the peripheral location of the infiltrate and by the fact that the patient was not immunosuppressed. The relative integrity of the lung defence mechanisms could have limited the bacterial burden, thus making the isolation of bacteria from sputum and bronchial aspirate difficult.

Although Horner's syndrome was not present, the characteristic nature and distribution of the pain indicate involvement of the lower brachial plexus root, probably due to irritation secondary to pleural inflammation. The patient was otherwise healthy, except for a moderate obstructive ventilatory defect attributable to his smoking habit. Interestingly, he had had no contact with animals.

Taking into account the clinical picture, the absence of neoplasm, the growth in pure culture of $P$. multocida from transthoracic needle aspirate, and the resolution of the clinical picture with antibiotic therapy, there can be little doubt about the aetiological role of $P$. multocida in this case. The case reported emphasizes the need for 
accurate diagnosis of patients with lung apical masses causing Pancoast's syndrome before planning therapy. Although malignant disease is the primary cause of the syndrome, infectious aetiologies must be taken into account when a diagnosis of malignancy is not easily obtained.

\section{References}

1. Pancoast HK. Superior pulmonary sulcus tumor: tumor characterized by pain, Horner's syndrome, destruction of bone and atrophy of hand muscles. J Am Med Assoc 1932; 99: 1391-1396.

2. Fraser RG, Paré JAP, Paré PD, Fraser RS, Genereux GP. Neoplastic disease of the lungs. In: Diagnosis of Diseases of the Chest. Philadelphia, Saunders, 1989; pp. 1424-1431.

3. Hubbert WT, Rosen MN. Pasteurella multocida infection in man unrelated to animal bite. Am J Public Health 1970; 60: 1109-1117.

4. Weber DJ, Wolfson JS, Swartz MN, Hooper DC. Pasteurella multocida infections: report of 34 cases and review of the literature. Medicine 1984; 63: 133-154.

5. Beyt BE, Sondag J, Roosevelt TS, Bruce R. Human pulmonary pasteurellosis. J Am Med Assoc 1979; 242: $1647-1648$.

6. Stathatos C, Kontaxis NA, Zafiracopoulos P. Pancoast's syndrome due to hydatid cyst of the thoracic outlet. $J$ Thorac Cardiovasc Surg 1969; 58: 764-768.

7. Simpson FG, Morgan M, Cooke NJ. Pancoast's syndrome associated with invasive aspergillosis. Thorax 1986; 41: 156-157.

8. Collins PW, de Lord C, Newland AC. Pancoast's tumour due to aspergilloma. Lancet 1990; 336: 1595.

9. Shamji FM, Leduc JR, Bormanis J, Sachs HJ. Acute Pancoast's syndrome caused by fungal infection. Can J Surg 1988; 31: 441-443.

10. Silverman MS, MacLeod JP. Pancoast's syndrome due to staphylococcal pneumonia. Can Med Assoc J 1990; 142: 343-345.

11. Gallagher KJ, Jeffrey RR, Kerr KM, Steven MM. Pancoast's syndrome: an unusual complication of pulmonary infection by Staphylococcus aureus. Ann Thorac Surg 1992; 53: 903-904. 